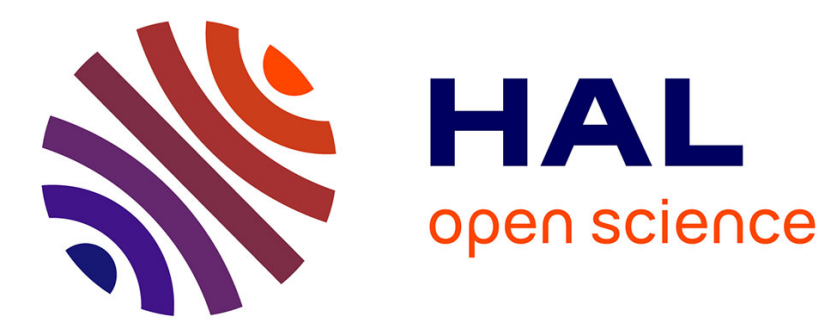

\title{
INTERNAL FRICTION AT MEDIUM TEMPERATURES IN HIGH PURITY ALUMINIUM
}

\author{
M. Nó, C. Esnouf, G. Thollet, J. San Juan, Gilbert Fantozzi
}

\section{To cite this version:}

M. Nó, C. Esnouf, G. Thollet, J. San Juan, Gilbert Fantozzi. INTERNAL FRICTION AT MEDIUM TEMPERATURES IN HIGH PURITY ALUMINIUM. Journal de Physique Colloques, 1987, 48 (C8), pp.C8-161-C8-166. 10.1051/jphyscol:1987821 . jpa-00227125

\section{HAL Id: jpa-00227125 https://hal.science/jpa-00227125}

Submitted on 1 Jan 1987

HAL is a multi-disciplinary open access archive for the deposit and dissemination of scientific research documents, whether they are published or not. The documents may come from teaching and research institutions in France or abroad, or from public or private research centers.
L'archive ouverte pluridisciplinaire HAL, est destinée au dépôt et à la diffusion de documents scientifiques de niveau recherche, publiés ou non, émanant des établissements d'enseignement et de recherche français ou étrangers, des laboratoires publics ou privés. 


\title{
INTERNAL FRICTION AT MEDIUM TEMPERATURES IN HIGH PURITY ALUMINIUM
}

\author{
M.L. Nó, C. ESNOUF* , G. THOLLET* J. SAN JUAN and G. FANTOZZI* \\ Dpto de Física del Estado Sólido. Facultad de Ciencias. \\ Universidad del Pais Vasco, Aptdo 644, SP-48009 Bilbao, Spain \\ "GEMPPM-LA 341, INSA, Bât. 502, F-69621 Villeurbanne Cedex. \\ France
}

Resumé.- Afin de déterminer le mécanisme qui est à $l$ 'origine des relaxations $P_{1}$ et $P_{1}$ nous avons comparé les résultats expérimentoux de frottement intérieur et de mieroscopie électronique d'un Al $99.9999 \%$, obtenus autour de $0.5 T^{\prime}$, avec les prévisions théoriques des différents modeles proposés dans la littérature.

Abstract. - In order to determine which mechanism is at the origin of the $P_{1}$ and $P_{1}$ relaxations, the internal friction and electron microscopy experimental results of $99.9999 \%$ Al around $0.5 T_{m}$ have been compared to the theoretical forecasts of different proposed models.

\section{1.- INTRODUCTION}

Under certain experimental conditions we can observe in the highest purity aluminium (99.99998) the $\mathrm{P}_{1}$ and $\mathrm{P}_{\bar{j}}$ relaxations around $0.5 \mathrm{~T}_{\mathrm{m}}\left(\right.$ at $\left.1 \mathrm{H}_{z}\right) / 1,2 /$. In a lower purity aluminium $(99.99998+10 \mathrm{ppm} \mathrm{Cu}$ or $\mathrm{Ag})$ we can observe a relaxation at higher temperatures which could correspond to $P_{1}$ relaxation of Al $99.99998(6 \mathrm{~N}) / 3 /$. Another kind of results, which we will discuss later on, have been obtained by kê et al. on $6 \mathrm{~N}$ Al $/ 4 /$.

In this paper we will try and analyse which model of all those proposed up to now coincides with the experimental behaviour of $P_{1}$ and $P_{1}^{i}$ relaxations.

\section{2.- EXPERIMENTAL CONDITIONS}

In this work, we have used a $6 \mathrm{~N}$ Al refined by zone melting. Some samples had been doped with $10 \mathrm{ppm}$ of $\mathrm{Cu}$ or Ag. All of them have been produced by CECM of Vitry/ Seine, France. These samples have undergone various thermo-mechanical treatments in order to create different microstructures : - cold rolled at room temperature (RT) and at 77K. - Torsional deformation at RT and at 77K. - Creep at $2009 \mathrm{C}$ and $3000 \mathrm{C}$. - Annealing at 600K.

Internal Friction (IF) spectra have been taken with an inverted torsion pendulum oscillating around $1 \mathrm{~Hz}$ with $2 \times 10^{-7}<\varepsilon_{\mathrm{m}}<5 \times 10^{-5}$ and $6 \mathrm{~K}<\mathrm{T}<730 \mathrm{~K}$. We can also superimpose a static torsional stress $\left(\sigma_{s}\right)$ upon the oscillating stress $\left(\sigma_{m}\right)$.

The microstructures of the samples has been analysed before and after applying these thermo-mechanical treatments, thanks to a JEOL $-200 \mathrm{CX}\left(\Delta \mathrm{V}_{\text {work }}=160 \mathrm{Kv}\right)$. The study focuses on those parts of the samples where the thickness is over 6000A so that they are representative of the bulk.

\section{3.- EXPERIMENTAL RESULTS}

* The samples deformed by torsion or cold-rolling at RT up to 508 show two relaxations $P_{1}$ and $P_{1}$ which are stable after a fixst annealing at $600 \mathrm{~K}$ (Fig.1 curve a). These relaxations are not affected by a static stress $\left(\sigma_{s}\right)$ superimposed upon the oscillating stress $\left(\sigma_{m}\right)$ and whose order of magnitude is up to 10 times $\sigma_{m}(F i g .2)$ $11,2,5 /$. The strengths of these relaxations show a maximum versus the oscillating amplitude (fig.3) /1,2,5/ while at the same time the peak shifts towards low temperatures. The activation energy of the $P_{1}$ relaxation was measured by two different ways : a) In a low frequency pendulum $/ 3 /$ b) By microcreep at various temperatures, using Klam's technique /2/. 
We obtained the following results : $\mathrm{H}_{\mathrm{P}_{1}}=1.1 \mathrm{eV}, \mathrm{H}_{\mathrm{P}_{1}}=0.8 \mathrm{eV}$.

By microcreep measurements and for different applied stress we have determined the activation volume of the process : $v_{P_{1}} \cong v_{P_{i}} \cong 1000 b^{3}$.

The observation of the samples by Transmission Electron Microscopy indicates that the dislocations are organised in cells formed by tangled dislocations (Fig.1a).

* The samples which have undergone creep through tension or torsion at $200 \mathrm{OC}$ or 300 \% do not show $P_{1}$ and $P_{i}$ relaxations; however they show a strong background towards high temperatures (Fig.l curve b) which corresponds to the low temperature side of a relaxation placed near the melting temperature $/ 2,5 /$. In this case, the samples show a polygonized microstructure (Fig.1b). The size of the subgrains is about 30 $\mathrm{\mu m}$ and the subboundaries are generally formed by families of dislocations that do not verify Frank's law.

* A deformation of at least 208 by torsion at RT, or a similar deformation by rolling at RT causes the reappearance of $P_{1}$ and $P_{i}$ relaxations. A latex creep makes them disappear again $/ 2,6 /$.

We could then conclude that $P_{1}$ and $P_{i}$ relaxations are associated to a deformation cells microstructure, while their disappearance is linked to a polygonized microstructure.

Experimental results on $99.99998 \mathrm{Al}+10 \mathrm{ppm}$ of $\mathrm{Cu}$ or $\mathrm{Ag}$ have been reported in $/ 3 /$ and could be summarized as follows :

There is only one relaxation which occurs at higher temperature $(\mathrm{T}(\mathrm{Al}+\mathrm{Ag})=526 \mathrm{~K}$, $T(A 1+C u)=565 K)$ than the $P_{1}$ relaxation, whose activation energy is $1.27 \mathrm{eV}$ for $\mathrm{Al}+\mathrm{Ag}$ and $1.54 \mathrm{eV}$ for $\mathrm{Al}+\mathrm{Cu}$. On the other hand a superimposed static stress does not affect internal friction spectra, and the internal friction maximum decreases versus oscillating amplitude. Furthermore, it is important to remark that the relaxation dissappears by creep.

\section{4.- DISCUSSION}

In this section, we will try to bring together all the models proposed in order to account for the relaxations that appear around $0.5 \mathrm{~T}_{\mathrm{m}}$ in aluminium and other f.c.c. metals. We will also take into account all the models proposed to explain creep in stage II, since the temperature range in which such stage occurs, as well as the activation energy and the activation volume either coincide or are close to those of $P_{1}$ and $P_{i}$ relaxation. So, we will analyse each model predictions, and will compare them to our experimental results.

a) Mechanisms connected with boundaries

Kê et al. /4/ have worked on $A l 6 \mathrm{~N}$ and they have noticed that in the case of polycrystals there is a relaxation at $480 \mathrm{~K}$ which they identify with Kê's peak and they attribute to the intercrystalline sliding at boundaries. If the grain size $\phi$ verifies $\phi>e$ (e being the sample's thickness), then the peak is replaced by another that they call "macrocrystalline peak" ( $T \cong 500 \mathrm{~K})$, attributable to a mechanism in which polygonization and boundaries play important roles. A 0.48 deformation on the previous sample makes the macrocrystalline peak disappear, and makes the $\mathrm{P}_{1}$ peak appear at $450 \mathrm{~K}$.

We have previously proved $/ 2,6 /$ that the background and $p_{1}$ relaxation depend on the thermomechanical treatments and the oscillation amplitude. In this way we can obtain variations up to $60 \mathrm{~K}$ in the peak temperature, depending on the experimental conditions. This makes us think that the three peaks mentioned by kê et al./4/ that have never been noticed at the same time, are the same $p_{1}$ relaxation. Furthermore, we have proved that both the appearance or disappearance of $P_{1}$ and $P_{1}$, as well as their intensity are independent from the grain size. This makes us think that $P_{1}$ and $P_{i}$ can in no way be intexpreted as a mechanism associated only with the boundaries as was formerly proposed by woirgard $/ 7 /$, who noticed $P_{1}, P_{2}$ and $P_{3}$ relaxations in $5 \mathrm{~N} A \mathrm{~A}$ both in monocrystals and polycrystals.

b) Cross-slip on planes \{111\}

Among the models proposed to account for cross-slip Friedel-Escaig's stands out $/ 8 /$. Let us start from a constriction which has nucleated on a fault ribbon 
spread on the primary slip plane of a f.c.c. crystal. Cross-slipping on another plane occurs when the two halves of the constriction are separated along the line, allowing some dislocationslength in between to be bowed out and to dissociate in the cross-slip plane as soon as the halves are brought apart (Fig.4). For some critical separation, $A B$, the configuration energy goes through a maximum, beyond which $A$ and $B$ are pushed apart under the work of stress, making the whole dislocation crossslip. The activation energy falls between $U_{C}$ and $2 U_{C}\left(U_{C}\right.$ being the constriction energy). If we consider that $U_{c}$ is of order of a jog creation energy $\left(U_{c}=0.42 e V\right)$ we obtain that the activation energy of this process is very inferior to that of $P_{1}$, $P_{1}$ and $P$ relaxations, so this process can not explain these relaxations.

The model proposed by Caillard $/ 9 /$ and later generalised by Morris $/ 10 /(F i g .5)$ assumes that in the case of three dislocations which come to an intersection, their corresponding burcers vectors are coplanar This model explained before in /5/ was proposed to explain the extraction of dislocations out of the polygonization walls being these formed by three families and we think that it could also be generalised to the deformation cells every time we have three coplanar vectors. Caillard shows that the stress that this mechanism needs to take place is very high (approximately 258 lower than orowan's stress). Such stress can not be justified by means of the stress applied in IF measurements; thus we consider that this process can not be responsible for $P_{1}$ and $P_{i}$ relaxations. On the other hand, the fact that the applied static stxess superimposed upon the oscillation stress does not affect $P_{1}$ and $P_{1}$ relaxations, means that this mechanism does not explain these relaxations $/ 2,5,6 /$. Any applied static stress higher than the oscillation stress should produce the cross-slip and then, internal friction should disappear. Furthermore, the activation energy for this process is weak if we consider that the constriction nucleation energy is more or less the same as the fog formation energy $\left(U_{C}=0.42 \mathrm{eV}\right)$.

Later on, Moxxis et al. /10/ observed that the extraction in the case of the three dislocations being on the same plane as the burgers vector, can take place through a simple slipping mechanism that needs climbing. We therefore think that in that case it is the climbing that controls the extraction mechanism.

\section{c) Slip on non-compact planes}

The experiments carried out by carrard $/ 11 /$ on monocrystals deformed by creep or tension along [112] directions, show that in the case of creep, the slipping on $\{100\}$ can be noticeable from 180 oC onwards for $10^{-7} s^{-1}<\varepsilon<10^{-5} s^{-1}$, whereas in the case of tension tests, it does not appear until 279 oc for $\varepsilon=12 \times 10^{-5} \mathrm{~s}^{-1}$.

There are several models proposed to account for slipping on non-compact planes. Among these, the appropriate one to explain the experimental results obtained up to this moment is glide on non-compact planes of dissociated dislocations on $\{111\}$. Such mechanism proposed by Friedel and later improved by Escaig / $12 /$ assumes a dissociated dislocation on a plane (111) that joins together along a critical length $A B$, producing double kinks on a non-compact plane, and then it dissociates again on a plane (111) (Fig.6). This mechanism needs to create four constrictions and two dissociated segments on a non-compact plane. So, the activation energy will be : $2 \mathrm{U}_{\mathrm{K}}=4 \mathrm{U}_{\mathrm{C}}+2 \mathrm{U}_{\mathrm{R}}$, $\mathrm{U}_{\mathrm{K}}$ being $\mathrm{kink}$ nucleation energy, $\mathrm{U}_{\mathrm{C}}$ constriction nucleation energy and $U_{R}$ the energy needed to create a dissociated segment $A A^{-}$or $B B$ : If we consider. the fact that $U_{C}$ can be compared with the jog creation energy, and that $U_{K}$ is between 0.9 and $1.13 \mathrm{eV} / 11 /$, the result is $1.3 \mathrm{eV}<2 \mathrm{U}_{\mathrm{K}}<1.7 \mathrm{eV}$. The activation energy seems to be slightly high compared with the activation energy of $P_{1}$ and $P_{1}$ relaxations, noticed on $6 \mathrm{~N} \mathrm{AI.} \mathrm{On} \mathrm{the} \mathrm{other} \mathrm{hand,} \mathrm{the} \mathrm{activation} \mathrm{volume} \mathrm{expected} \mathrm{for} \mathrm{this}$ mechanism is between $350 \mathrm{~b}^{3}<\mathrm{v}<700 \mathrm{~b}^{3} / 11 /$, a value' which is slightly low compared with the activation volume measured in our micro-creep tests $900 \mathrm{~b}^{3}<\mathrm{v}<1600 \mathrm{~b}^{3}$ $/ 1 /$. We must also point out that such mechanism should give an IF peak similar to Bordoni's. Therefore we could expect non linear effects like those described by Esnouf $/ 13 /$ for Bordoni relaxation. Thus, in the case of weak internal stress, both the internal friction peak strength $\delta_{M}$ and its correspondent temperature must have a maximum versus $\sigma_{m}$. So, $\delta_{M}$ and $T_{M}$ increase as Pare's condition is not fulfilled, in such a way that if we apply a static stress superimposed upon the oscillating stress, the strength of the relaxation will increase. Our experimental results show a maximum $\delta_{\mathrm{H}}$ versus $\sigma_{\mathrm{m}}$ but on the contrary $\mathrm{T}_{\mathrm{m}}$ decreases with a non linear.dependency versus $\sigma_{m}$,while $\sigma_{s}$ does not affect $p_{1}$ and $p_{1}$ relaxations, as opposed to the theoretical predictions. 
Al samples doped with $10 \mathrm{ppm}$ of $\mathrm{Cu}$ or Ag have values for the activation energy obtained for $P$ relaxation and IF results $/ 3 /$ that can be explained through FriedelEscaig"s mechanism. However the study will be incomplete until the microstructure of the samples is throughly analysed and internal friction is studied in detail in order to precise the influence of the applied tension. As we will see later on, the results obtained up to now also coincide with a dislocation glide mechanism controlled by jog climb.

If, instead of considering free dislocations we considered a subboundary with three families of dislocations verifying Frank's law, then one of the three families is screw. Under stress, mixed dislocations can glide on two planes $\{111\}$ while screw dislocations can do it on $\{100\}$ or on $\{110\}$ after a Friedel-Escaig mechanism in which only one kink will be nucleated since node 0 is able to glide freely (Fig.7)/15/. The activation energy expected will be : $0.65 \mathrm{eV}<\mathrm{U}_{\mathrm{K}}<0.85 \mathrm{eV}$, which is too low to account for $P_{1}$ relaxation. Moreover, we have noticed through our experiments that $P_{1}$ relaxation disappears from polygonized samples, which seems to indicate that this mechanism is not the cause of $P_{1}$ relaxation. On the other hand, it seems unlikely that in the case of non polygonized samples with a tangled dislocation structure there is a high number of screw dislocations that cause a relaxation as intense as $\mathrm{P}_{1}$ or $\mathrm{P}_{1}$. Nevertheless a more thorough analysis of the microstructure would be advisable to verify this point.

\section{d) Slip controlled by pipe-diffusion}

Let us suppose there is a screw or mixed dislocation with a sessile jog. The dislocation cannot glide uniess the jog climbs thanks to a non conservative movement which provides vacancies created either in the bulk or near the dislocation (Fig.8!. In this process, the activation energy is around the auto-diffusion energy ( $\left.H_{v}=1.5 e v\right)$ or the pipe -diffusion $\left(H_{d}=1.1 \mathrm{eV}\right)$, and it depends slightly on the type of dislocation $/ 14 /$. In such conditions $\mathrm{H}_{\mathrm{v}}$ is too high to be responsible for the relaxations we axe dealing with. However, relaxations $P_{1}$ and $P_{i}$ can be explained in terms of pipe-diffusion. In this mechanism, a static stress superimposed upon $\sigma_{\mathrm{m}}$ cannot affect the relaxation because it will only change the oscillation centre of the dislocation line. This prediction agrees with the experimental results. On the other hand, from the value $\sigma_{m}$ we could expect a decrease in the internal friction maximum versus the oscillating stress. For high stress, the number of vacancies absorbed or emitted during half cycle of the oscillating stress is limited by the number of vacancies, $\mathrm{N}_{\mathrm{v}}$, that may be created near a jog at a given temperature. Therefore, the number of jumps $N$ that the dislocation may have, amount that must be increased with oscillating stress, will be limited by this process. Thus, the energy dissipated in a cycle (at a given temperature) stays the same whereas the elastic energy increases with stress and as a consequence the maximum of internal friction decreases with stress. This behaviour agrees with the experimental results (see Fig.3) $/ 2,6 /$.

On the other hand, we could expect that in the case of polygonized samples, the dislocations are more straight (the polygonization walls are flat to minimize the energy) and therefore there will hardly be any deformation jogs and so, they would have to be created. In this case the activation energy would correspond to that of a double jog nucleation, which is very high $/ 5 /$.

In this way, this mechanism seems capable of accounting for all results concerning relaxations $P_{1}$ and $P_{j}$ in $6 \mathrm{~N}$ Al. For $6 \mathrm{~N}$ Al doped with Ag or Cu, the experimental results obtained in internal friction can also be explained in terms of this mechanism. Indeed, in this case, the impurities will tend to place on the jogs modifying the climb energy of the jog. Therefore the activation energy of the process must be slightly higher than that of pipe-diffusion, in agreement with the experimental results.

\section{5.- CONCLUSIONS}

Considering our experimental results and the models proposed so far, we can conclude that the only mechanism capable of accounting for $P_{1}$ and $P_{1}$ relaxations in $6 \mathrm{~N} \mathrm{Al}$ is dislocation glide (screw for $\mathrm{P}_{1}$, mixed for $\mathrm{P}_{j}$ ) controlled by jog climb and vacancy pipe-diffusion. However, for $6 \mathrm{~N}$ Al doped with impurities, we need some supplementary internal friction tests and a characterization of the samples microstructure in order to state if the observed $P$ relaxation is the same as $P_{1}$ relaxation which has shifted towards high temperatures due to the presence of impurities, or 
if this relaxation is caused by another kind of process.

\section{REFERENCES}

1 .- M.L.Nó, J.San Juan, C.Esnouf, G.Fantozzi, A.Bernalte; J. de Phys.44(1983)C9-751

2 .- M.L.Nó; Thesis University of Lyon (France) (1985)

3 .- C.Esnouf, A.Kulik, J.F.Theumann,M.L.Nó; This conference

4 .- T.S.Kê, P.Cui, S.C.Yan, Q.Huang; Phys.Stat.Sol. (a) 86 (1984) 593

5 .- M.L.Nó, C.Esnouf, J.San Jlian, G.Fantozzi; J. de Phys. 46 (1985) C10-347

6 .- M.L.No, C.Esnouf, J.San Juan, G.Fantozzi; in press.

7 .- J.Woirgard; Phil.Mag. 33 (1976) 623

8 .- B.Escaig; J. de Phys. 35 (1974) C7-151

9 .- D.Caillard, J.L.Martin; Acta Met. 31 (1983) 813

10. - M.A.Morris, J.L.Martin; Acta Met 32 (1984) 549

11.- M .Carrard; Thesis University of Lausanne (1985)

12.- B.Escaig; Phys. Stat. Sol. 28 (1968) 463

13. - C.Esnouf; Thesis University of Lyon (1978)

14.- M.L.No, C.Esnouf, J.San Juan, G.Fantozzi; Scripta Met. 21 (1987) 213

15. - D.Caillard; Phil. Mag. A51 (1985) 157

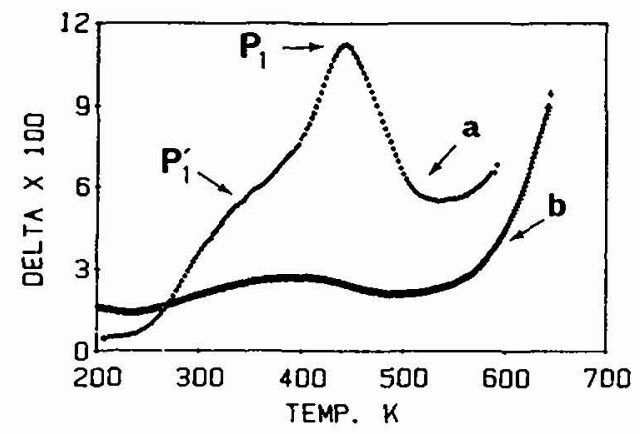

Fig.1.-

Internal Friction spectra for different microstructural states and their corresponding transmission electron micrographies $\left(\varepsilon_{\mathrm{m}}=8 \times 10^{-6}\right)$.

a) samples deformed by rolling or torsion at RT or at $77 \mathrm{~K}$.

b) Samples deformed by creep at 200 oc or at 300 oc.

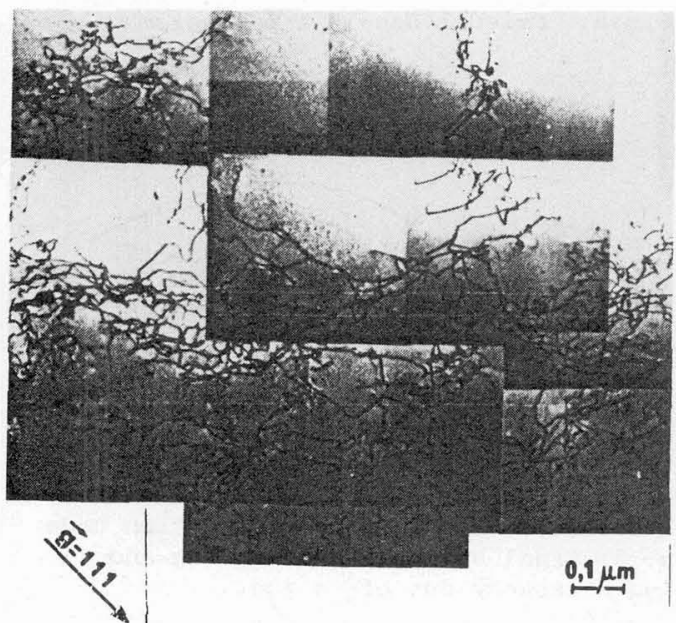

a.

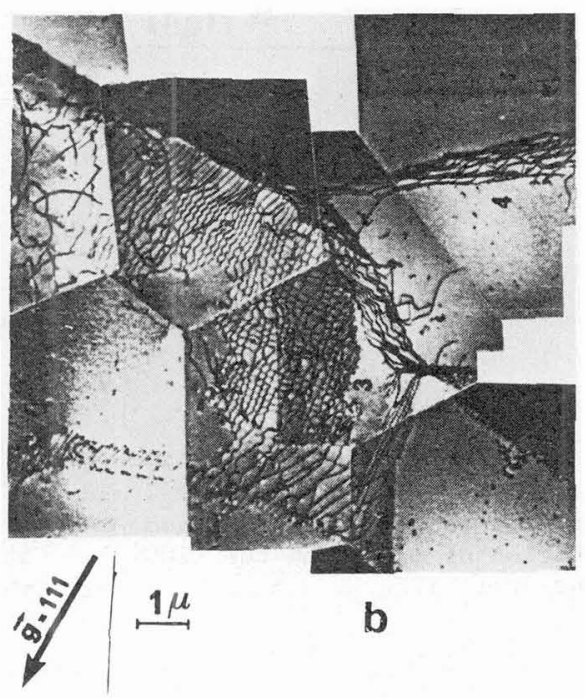




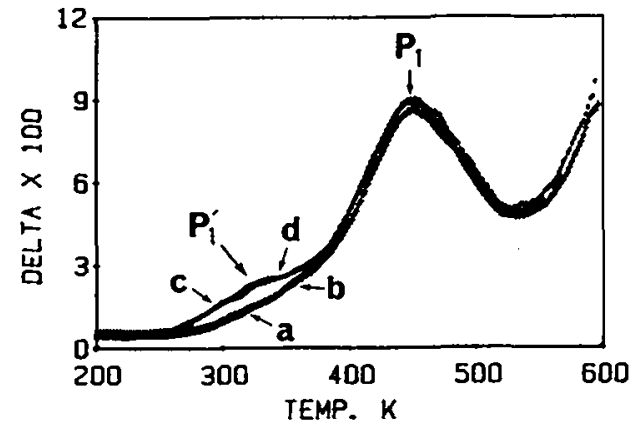

Fig.2. $-\sigma_{S}$ influence on Internal Friction spectrum. $\sigma_{m}=2 \times 10^{-6} \mu$, a) $\left.\sigma_{s}=0, b\right) \sigma_{s}=10^{-5} \mu$ c) $\left.\sigma_{s}=2 \times 10^{-5} \mu, d\right) \sigma_{s}=4 \times 10^{-5} \mu$

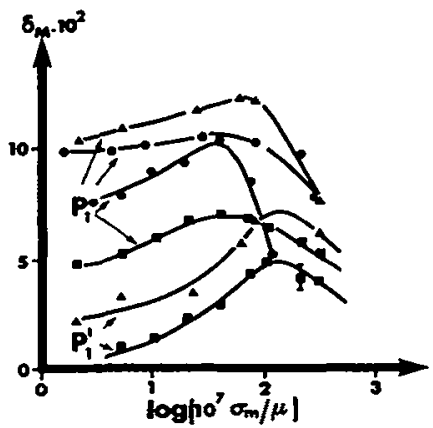

Fig.3.- Evolution of the $P_{1}$ and $P_{i}$ relaxations strength versus $\sigma_{\mathrm{m}}$ for different thermomechanical treatments $/ 2 /$.

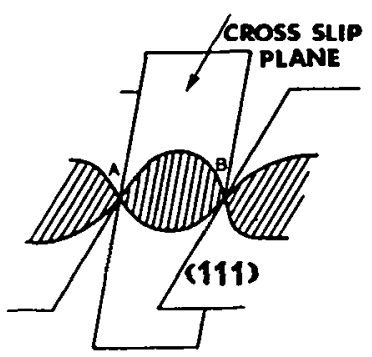

Fig.4.- Cross-slip in the Escaig's model.

Fig.5.- Cross-slip mechanism at the nodes of subboundaries : we can see the cross-slip of dislocation $3 / 9 /$.
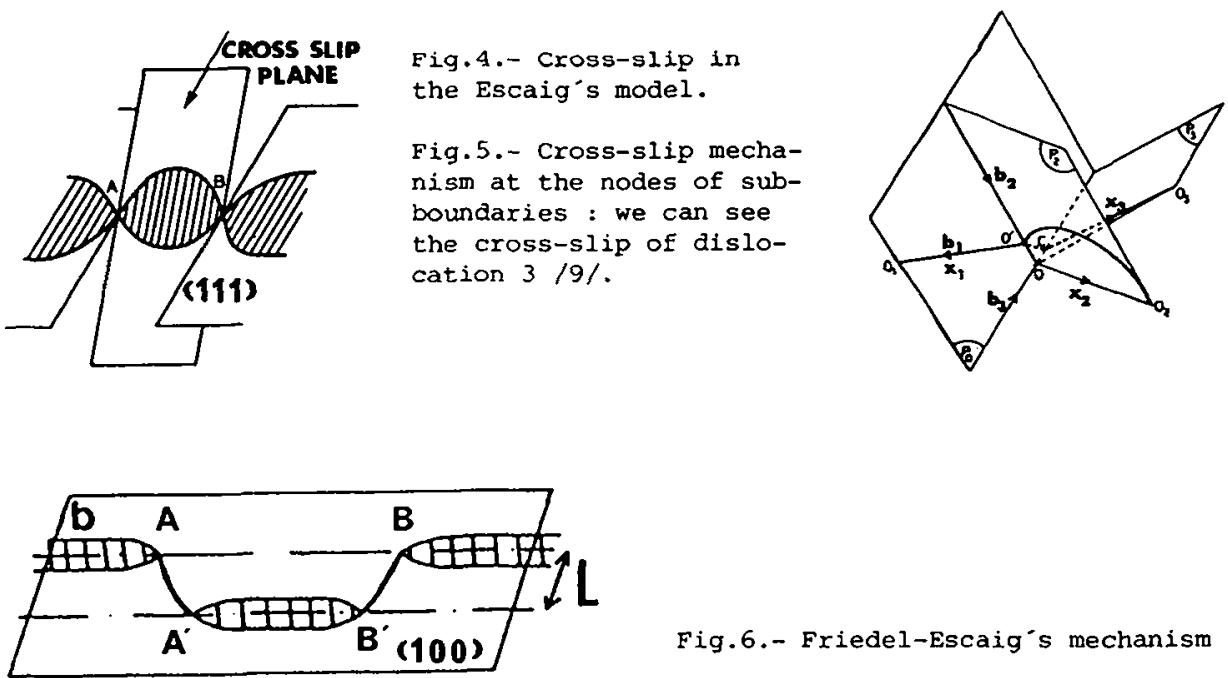

Fig.6.- Friedel-Escaig's mechanism /11/.

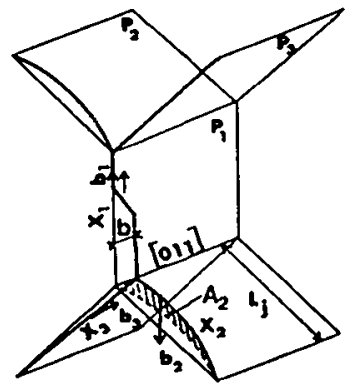

Fig.7.- Glide motion in $P_{1}, P_{2}$, and $P_{3}$ planes iue to nucleation and migration of a kink on the $\{100\}$ plane $\left(P_{1}\right) / 15 /$.

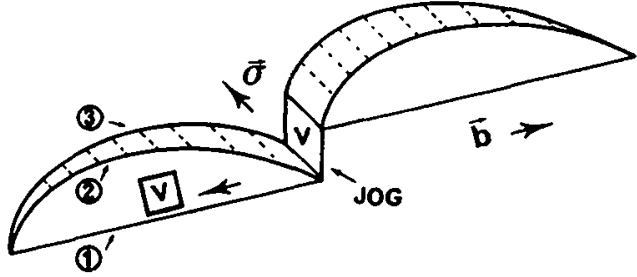

Fig.8.- Glide controlled by pipe-diffusion: 1) Initial state. 2) Loop of dislocation under stress. 3) Final state after creating and emitting a vacancy out of a jog. 